\title{
The dietary diversity and stunting prevalence in minority children under 3 years old: a cross-sectional study in forty-two counties of Western China
}

\author{
Anqi Wang ${ }^{1,2}$, Robert W. Scherpbier ${ }^{3}$, Xiaona Huang ${ }^{3}$, Sufang Guo ${ }^{4}$, Yuning Yang ${ }^{3}$, \\ Jonathan Josephs-Spaulding ${ }^{5}$, Chuyang $\mathrm{Ma}^{6}$, Hong Zhou ${ }^{1,7 *}$ and Yan Wang ${ }^{1,7}$ \\ ${ }^{1}$ Department of Maternal and Child Health, School of Public Health, Peking University, Beijing 100191, People's Republic of China \\ ${ }^{2}$ Department of Epidemiology, Johns Hopkins Bloomberg School of Public Health, Baltimore, MD 21205, USA \\ ${ }^{3}$ United Nations International Children's Emergency Fund China, Beijing 100600, People's Republic of China \\ ${ }^{4}$ United Nations International Children's Emergency Fund Regional Office for South Asia, Kathmandu, Nepal 44600 \\ ${ }^{5}$ Department of Environmental Health and Engineering, Johns Hopkins Bloomberg School of Public Health, \\ Baltimore, MD 21205, USA \\ ${ }^{6}$ Beijing Lu he International Academy, Beijing 101149, People's Republic of China \\ ${ }^{7}$ Environmental and Spatial Epidemiology Research Center, National Human Genetic Resources Center, Beijing 100191, \\ People's Republic of China
}

(Submitted 22 February 2017 - Final revision received 2 September 2017 - Accepted 13 September 2017)

\section{Abstract}

As a key indicator of childhood malnutrition, few studies have focused on stunting in relation to various socio-economic factors in which disadvantaged groups face in China. We conducted a community-based cross-sectional study incorporating forty-two rural counties in seven western provinces of China in 2011. In total, 5196 children aged 6-23 months were included. We used Poisson regression to examine risk factors for inadequate minimum dietary diversity (MDD) and stunting status, respectively. Overall, the proportion of children not meeting MDD was 44.5\%. Children aged 6-11 months (adjusted risk ratio (ARR) $=1.39 ; 95 \%$ CI 1.31, 1.49), with two siblings (ARR $=1.09 ; 95 \%$ CI 1.02, 1.17), delivered at home $(\mathrm{ARR}=1 \cdot 30 ; 95 \% \mathrm{CI} 1 \cdot 20,1 \cdot 41)$, within $\mathrm{Yi}(\mathrm{ARR}=1 \cdot 15 ; 95 \% \mathrm{CI} 1 \cdot 04,1 \cdot 28)$ or Uighur groups (ARR $=1 \cdot 52 ; 95 \% \mathrm{CI} 1 \cdot 36,1 \cdot 71)$, with an illiterate caregiver ( $\mathrm{ARR}=2 \cdot 12 ; 95 \% \mathrm{CI} 1 \cdot 52,2 \cdot 96$ ), receiving lowest income (ARR $=1 \cdot 32 ; 95 \%$ CI $1 \cdot 17,1 \cdot 50)$, and with breast-feeding in the last day $(\mathrm{ARR}=1.55 ; 95 \% \mathrm{CI} 1.44,1.66)$ were more likely to have inadequate MDD. Moreover, inadequate MDD was positively associated with stunting (ARR $=1 \cdot 15 ; 95 \%$ CI 1.01, 1.31). Other determinants for stunting were age, sex, place of delivery, minority group and income. The stunting prevalence and proportion of inadequate MDD remained high in Western China; to reduce stunting rates of ethnic minorities, further efforts addressing appropriate dietary feeding practices are needed, especially within these groups.

\section{Key words: Dietary diversity: Complementary feeding: Stunting prevalence: Minority groups}

In past decades, monumental progress has been made on under5 year child mortality reduction in China. The under-5-childrenmortality rate was mitigated by $78 \%$, from $79 \cdot 2 / 1000$ live births in 1991 to $10 \cdot 7 / 1000$ live births in 2015; this improvement led the country to achieve the Millennium Development Goal 4 ahead of schedule ${ }^{(1,2)}$. In addition to substantial improvement regarding childhood survival status, there has been a significant increase of attention by both the government of China and the global community in regard to achieving early childhood development goals over the past years ${ }^{(3,4)}$. Globally there are over 250 million children, of that number the 17.43 million that live in China were estimated to be unable to fulfil their full potential due to multiple adversities which can be marked by the lack of adequate nutrition, poor health or stimulation, inadequate nurturing, and perilous environments according to the newly released Lancet series ${ }^{(5,6)}$.

Maternal and child nutrition status plays an important role in infant morbidity, early development and even long-term health; childhood stunting is the foundational cause of poor cognition, low adult wages and lost productivity later in life ${ }^{(7)}$. The 65 th World Health Assembly set a target that by the year of 2025, the stunting prevalence of children under 5 years should be mitigated by $50 \%$ as compared with the baseline in 2010 established by the World Health Organization ${ }^{(8)}$. Globally, approximately 171 million children under the age of 5 years had some sort of stunting that affected their development ${ }^{(9)}$. Previous studies have shown that the stunting prevalence inequality among different populations is attributed to social, economic and

Abbreviations: MDD, minimum dietary diversity; RR, risk ratio.

* Corresponding author: H. Zhou, fax +86106202 3133, email hongzhou@bjmu.edu.cn 
political determinants ${ }^{(7,10)}$. Among all risk factors, feeding practices has the most direct impact upon childhood stunting. More so, appropriate interventions on the modification of feeding practices has been proved to be effective ${ }^{(11,12)}$.

The significant inequities on under 5-year child survival situations among different regional populations and ethnic minority groups in China have been recognised at the national level $^{(1)}$. Some regional studies report that the stunting rate among minorities was higher than the Han's, which is the major ethnic group in China ${ }^{(13-16)}$. In addition to the socio-economic disparities, ethnic minorities have various culture and religious beliefs; therefore, these fundamental roles may dictate their feeding patterns and which may differ from the Han's dietary practices. For example, a previous study within Tibet suggested that unbalanced diets may be linked to a greater stunting prevalence in Tibetan children living at a higher altitude ${ }^{(17)}$. Moreover, Uygur mothers were unlikely to feed their children breast milk exclusively within the first 6 months; this is due to the traditional belief that breast milk was inadequate for the growth of children ${ }^{(18)}$. Tibetan mothers were also inclined to introduce water early as following their ethnic tradition ${ }^{(19)}$. Although some studies have reported breast-feeding practices of the Chinese ethnic minorities, limited studies focused on complementary food diversity of children and the influence of different dietary patterns on the growth of children. To further support interventions on mitigating stunting in poor and remote minority areas, it is first necessary to understand the current prevalence of stunting and its numerous risk factors. Therefore, we conducted a population-based study in forty-two rural counties in the western provinces of China; these areas accounted for most of China's ethnic minorities as well as the most resource limited region in China. Our study methods were designed to identify the association between complementary dietary diversity and infant stunting, especially the impact for children of various ethnic groups and their respective varying dietary patterns.

\section{Methods}

\section{Study design and setting}

This study was a cross-sectional baseline survey on feeding practices and child growth in 2011, covering forty-two rural counties in seven western provinces of China (Gansu, Qinghai, Xinjiang, Sichuan, Guizhou, Yunnan and Tibet), as part of maternal and child health $(\mathrm{MCH})$ programme funded by UNICEF and the National Health And Family Planning Commission Of China (NHFPC). Counties were selected by NHFPC and UNICEF as programme counties due to their poor socio-economic development in addition to low $\mathrm{MCH}$ performance. Under-5 mortality rates were higher in surveyed counties (up to $21 \cdot 1 / 1000$ live births) as compared with the national average of $16 \cdot 4 / 1000$ live births in 2010; the per capita income of $3239 \mathrm{RMB}$ within these forty-two counties were below the national average 5919 RMB in $2010^{(20)}$. The Ethical Committee of Peking University Health Science Centre approved this study.

A multistage sampling method was employed to select townships and villages in each county. First, fifteen administrative villages per county and two nature villages per administrative village were selected at random with population proportional to size. Within each selected nature village, ten households with children under 3 years of age were selected according to a full registration list for each village by simple random sampling; the list of children was provided by local village doctors. Random numbers for selection criteria were generated by a random number table, and staff members of this programme completed all the sampling processes. In sparsely populated Tibet, only two or three households with children under 3 years were randomly sampled and selected in each nature village. The youngest children and their caregivers in each selected household were interviewed face-to-face.

Because this study was designed to collect relevant baseline data for a childhood intervention project, we expected to see a decrement in stunting prevalence in the final evaluation. The following formula was used to calculate the sample size:

$$
n=2 \overline{\mathrm{pq}}\left(Z_{\alpha}+Z_{\beta}\right)^{2} /\left(p_{1}-p_{0}\right)^{2}, \overline{\mathrm{p}}=\left(p_{1}+p_{0}\right) / 2, \overline{\mathrm{q}}=1-\overline{\mathrm{p}} .
$$

We used 0.05 as the statistical significance $(\alpha)$ and set the power of the test $(1-\beta)$ as $0 \cdot 8$. We assumed the baseline stunting prevalence $\left(p_{0}\right)$ would be $50 \%$ and expected a relative $20 \%$ decrement of stunting prevalence $\left(p_{1}-p_{0}\right) / p_{0}$. Finally, in consideration of a design effect of 2 and with an $80 \%$ response rate, the needed total sample size of children would be 1944 (972:972).

\section{Data collection}

A structured questionnaire for caregivers was provided in our survey. Items in the questionnaire were selected from 'Multiple Indicator Cluster Survey' manual published by $\mathrm{UNICEF}^{(21)}$. The dietary intake diversity assessment involved seven foods groups: (1) grains, roots and tubers, (2) legumes and nuts, (3) dairy products (milk, yogurt and cheese), (4) flesh foods (meat, fish, poultry and liver/organ meats), (5) eggs, (6) vitamin A-rich fruits and vegetables and (7) other fruits and vegetables. Data on dietary intake for the children $24 \mathrm{~h}$ before the survey were obtained through caregivers' recall. The questionnaire also included questions on the socio-economic characteristics of the household (ethnicity, education of the caregivers and income) and on the age, sex, birth order and place of delivery of the children.

Length (for children aged 0-23 months) and height (for children aged 24-35 months) were measured by two anthropometrists in each sampled village. Each measurement was performed twice and the average of both dimensions were used in the analysis. All anthropometrists underwent a systemic standardised anthropometric training before the study and followed the unified anthropometry manuals for consistent results. All village study sites used the same high-precision measuring equipment. Length was measured to the nearest $0 \cdot 1 \mathrm{~cm}$ with the WB-B Length Meter (Wujin Weighing Apparatus Factory). Height was measured to the nearest $0 \cdot 1 \mathrm{~cm}$ with the $\mathrm{SH}-2 \mathrm{~B}$ Stadiometer (Wujin Weighing Apparatus Factory). Data collection was monitored for quality control by the Peking University/UNICEF team in fourteen counties and by staff from the local health bureau or the $\mathrm{MCH}$ hospitals in twenty-eight counties. All data collected were entered as dual replicates into a database. 


\section{Statistical analysis}

WHO 2006 Child Growth Standard, length/height-for-age $z$ scores (HAZ) was used as an evaluation standard of stunting status as recommended by $\mathrm{WHO}^{(22)}$. All children with $z$ score of HAZ below 2 were defined as stunted within our study. The minimum dietary diversity (MDD) was selected from 'Indicators for assessing infant and young child feeding practices' published by WHO in 2008 as a core indicator in our study for accessing complementary feeding practice ${ }^{(23)}$. WHO guidelines implied that MDD was defined as 'receiving foods from 4 or more food groups $24 \mathrm{~h}$ previous to the survey was given' ${ }^{,(23)}$. We also use the term 'inadequate MDD' to refer to children who did not achieve the recommended MDD in the following context.

Within our study, thirty-two different ethnic groups were reported and ethnicity was categorised as either Han or other minority groups (Yi, Tibetan, Miao and other ethnics). Additional covariates include sex of children (male and female), age of the child, birth order of children (1,2, 3 or more), caregiver's education (illiterate, primary school, secondary school, college and above), place of delivery (county hospital, township hospital or at home), income (poorest, poor, middle, richer and richest) and breast-feeding during the previous day (yes/ no).

As the MDD index is generally targeted for infants under the age of 2 years, we restricted our study population to children aged 6-23 months for the final analyses ${ }^{(8)}$. Socio-economic characteristic distributions were calculated and compared between children within the Han and minority groups using chisquare tests. Socio-economic determinants of inadequate MDD and stunting were examined with Poisson regression; all were weighed for the variation in the sampling distribution of a population of children 6-23 months across counties. Both crude, adjusted relative risks and $95 \% \mathrm{CI}$ reports were adjusted for the child's sex, age, birth order, ethnicity of the caregiver, caregiver's education, the place of birth, income, breast-feeding during the previous day and MDD (only for stunting). We also calculated and compared the frequencies of each types of complementary foods consumptions among different ethnic groups and by different age groups. All analyses were conducted using STATA version 13.0

\section{Results}

\section{Samples description}

In our survey, a total of 8964 children aged under 5 years were interviewed. The present study included 5196 children aged 6-23 months; among them, 53.1\% were males and 53.8\% were the first baby for their parents. A total of $31.5 \%$ of children's caregivers were illiterate, and $23.8 \%$ children were delivered at their home, as shown in Table 1 . Among all children belonging to various proportions of ethnic groups there were $38.0 \%$ Han, $13.8 \% \mathrm{Yi}, 13.0 \%$ Tibetan, $8.6 \%$ Uighur and $26.5 \%$ belonged to other minority groups. The distributions of various age groups were similar in different ethnic groups $(P=0.234)$. Slight differences in child sex distribution showed variation between different ethnic groups $(P<0 \cdot 05)$. For caregiver's education, $68.7 \%$ of the $\mathrm{Yi}$ and $58.5 \%$ of the Tibetan groups were illiterate; in contrast, the prevalence of illiteracy was only $22 \cdot 2$ and $3 \cdot 4 \%$ among the Han and Uighur groups, respectively. Different ethnic groups also showed disparity based on the place of delivery. For example, $19.6 \%$ of the Yi children were delivered at a county level hospital, this proportion was higher in the Tibetan (49.9\%), Uighur (67.2\%) and Han children (73.1\%). Moreover, the income level in Han group had a relatively balanced distribution, whereas the other ethnic groups had more households belonged to the poorest or poor quintiles of income (Han: $37.0 \%$, Yi: $72.5 \%$, Tibetan: $50.0 \%$, Uighur: $48.8 \%$, $P<0.001)$. The Han group also had less of the proportion of breast-feeding during the previous day as compared with other minorities (Han: $37 \cdot 3 \%$, Yi: $65.5 \%$, Tibetan: $40 \cdot 1 \%$, Uighur: $55 \cdot 6 \%, P<0.001)$, as shown in Table 1.

\section{Minimum dietary diversity}

Table 2 showed the bivariate and multivariate regression output of risk factors associated with an inadequate MDD. Among children within the age groups of 6-23 months in our study, $44.5 \%$ of them did not achieve the MDD. Among all ethnic groups, the Yi minority had the poorest performance with $73.1 \%$ of children not reaching MDD. The Uighur and Tibetan groups also had a relatively high probability of not receiving the MDD in their daily meals, as compared with the Han children. The variations of inadequate MDD differed by age, birth order of children, caregiver's education level, place of delivery, income and breast-feeding during the previous day were also significant. Children aged 6-11 months had a higher proportion of inadequate MDD as compared with older children (58.6 $v$. $36 \cdot 1 \%$, adjusted risk ratio $(\mathrm{RR})=1 \cdot 39$ ). Children with a birth order of being third or greater had a higher proportion of the inadequate MDD in comparison to first born children (65.9 $v$. $38.2 \%$, adjusted $\mathrm{RR}=1 \cdot 07$ ). Children with illiterate caregivers had the highest probability of inadequate MDD (58.8\%) in comparison with other caregivers with higher education levels. In addition, children from the poorest quintile families who were delivered at home had a significant positive association with poor performance on dietary diversity, with adjusted RR of 1.32 and 1.30 , respectively. No significant difference of dietary diversity was found between male and female children.

Further analysis on types of complementary foods found that $91.2 \%$ of children received grains, roots and tubers during the previous day of survey, as shown in Fig. 1. More than half of children received dairy products $(57.4 \%)$, flesh foods $(54.2 \%)$, vitamin A-rich fruits and vegetables $(57 \cdot 1 \%)$ and other fruits and vegetables $(57.9 \%)$, respectively. Only $34.3 \%$ of children had eggs intake and only $15.9 \%$ of children received legumes and nuts. When comparing variation of these types of complementary foods by ethnic groups as compared with other, it was found that the Yi group had a lower consumption of every kind of complementary foods, especially dairy products (Yi $v$. Han $=19.5 v .62 .9 \%, P<0.001$ ), eggs (Yi $v$. Han $=25.1 v .43 .6 \%$, $P<0.001$ ), vegetables and fruits (Yi $v$. Han $=41.6 v .71 .8 \%$, $P<0.001)$. The Uighur ethnic also showed similar results with most types of complementary foods intake; the only exceptions were for flesh foods (68.5\%), and non-vitamin A-rich fruits and vegetables (61.6\%). Tibetan children tend to have a greater 
Table 1. Socio-demographic characteristics among children aged 6-23 months with Han and ethnic groups in forty-two counties in Western China (2011) (Numbers and percentages)

\begin{tabular}{|c|c|c|c|c|c|c|c|c|c|c|c|c|c|c|}
\hline \multirow[b]{3}{*}{ Characteristics } & \multicolumn{2}{|c|}{ Total } & \multicolumn{2}{|c|}{ Han } & \multicolumn{2}{|c|}{$\mathrm{Yi}$} & \multicolumn{2}{|c|}{ Tibetan } & \multicolumn{2}{|c|}{ Uighur } & \multicolumn{2}{|c|}{ Other } & \multirow[b]{3}{*}{$x^{2}$} & \multirow[b]{3}{*}{$P$} \\
\hline & \multicolumn{2}{|c|}{5196} & \multicolumn{2}{|c|}{1977} & \multicolumn{2}{|c|}{718} & \multicolumn{2}{|c|}{674} & \multicolumn{2}{|c|}{448} & \multicolumn{2}{|c|}{1379} & & \\
\hline & $n$ & $\%$ & $n$ & $\%$ & $n$ & $\%$ & $n$ & $\%$ & $n$ & $\%$ & $n$ & $\%$ & & \\
\hline Age of child (months) & & & & & & & & & & & & & 5.563 & 0.234 \\
\hline $6-11$ & 1938 & $37 \cdot 3$ & 706 & $35 \cdot 7$ & 271 & $37 \cdot 7$ & 253 & 37.5 & 162 & $36 \cdot 2$ & 546 & 39.6 & & \\
\hline $12-23$ & 3258 & $62 \cdot 7$ & 1271 & $64 \cdot 3$ & 447 & $62 \cdot 3$ & 421 & 62.5 & 286 & 63.8 & 833 & 60.4 & & \\
\hline Sex of child & & & & & & & & & & & & & $10 \cdot 362$ & $<0.05$ \\
\hline Male & 2747 & $53 \cdot 1$ & 1066 & 54.2 & 356 & $49 \cdot 6$ & 333 & $49 \cdot 4$ & 236 & 53.2 & 756 & $55 \cdot 1$ & & \\
\hline Female & 2429 & $46 \cdot 9$ & 901 & $45 \cdot 8$ & 362 & $50 \cdot 4$ & 341 & $50 \cdot 6$ & 208 & $46 \cdot 8$ & 617 & 44.9 & & \\
\hline Birth order of child & & & & & & & & & & & & & 817.350 & $<0.001$ \\
\hline 1 & 2698 & 53.8 & 1163 & 60.5 & 194 & $27 \cdot 1$ & 372 & 55.4 & 219 & 53.4 & 750 & 57.9 & & \\
\hline 2 & 1640 & $32 \cdot 7$ & 623 & 32.4 & 188 & $26 \cdot 3$ & 239 & 35.6 & 137 & 33.4 & 453 & $35 \cdot 0$ & & \\
\hline$\geq 3$ & 675 & 13.5 & 135 & $7 \cdot 0$ & 333 & $46 \cdot 6$ & 60 & 9.0 & 54 & $13 \cdot 2$ & 93 & $7 \cdot 2$ & & \\
\hline Caregiver's education & & & & & & & & & & & & & $1180 \cdot 136$ & $<0.001$ \\
\hline Illiteracy & 1632 & 31.5 & 438 & $22 \cdot 2$ & 492 & $68 \cdot 7$ & 394 & 58.5 & 15 & 3.4 & 293 & $21 \cdot 3$ & & \\
\hline Primary & 1452 & 28.0 & 590 & 29.9 & 155 & 21.6 & 181 & 26.9 & 103 & $23 \cdot 1$ & 423 & $30 \cdot 7$ & & \\
\hline Secondary & 1950 & 37.6 & 885 & 44.8 & 69 & $9 \cdot 6$ & 73 & $10 \cdot 8$ & 315 & $70 \cdot 6$ & 608 & $44 \cdot 2$ & & \\
\hline College and above & 152 & 2.9 & 61 & 3.1 & 0 & 0.0 & 26 & 3.9 & 13 & 2.9 & 52 & 3.8 & & \\
\hline Place of delivery & & & & & & & & & & & & & $1624 \cdot 145$ & $<0.001$ \\
\hline County hospital & 3135 & $61 \cdot 7$ & 1408 & 73.1 & 140 & $19 \cdot 6$ & 332 & 49.9 & 291 & $67 \cdot 2$ & 964 & $71 \cdot 7$ & & \\
\hline Township hospital & 714 & 14.6 & 282 & 14.6 & 21 & 2.9 & 77 & 11.6 & 123 & 28.4 & 238 & $17 \cdot 7$ & & \\
\hline At home & 1208 & $23 \cdot 8$ & 237 & $12 \cdot 3$ & 554 & 77.5 & 256 & 38.5 & 19 & 4.4 & 142 & $10 \cdot 6$ & & \\
\hline Postpartum visit & & & & & & & & & & & & & 258.694 & $<0.001$ \\
\hline Yes & 2072 & $42 \cdot 8$ & 843 & $45 \cdot 7$ & 112 & $16 \cdot 9$ & 269 & $43 \cdot 2$ & 151 & $36 \cdot 3$ & 697 & 53.9 & & \\
\hline No & 2767 & $57 \cdot 2$ & 1002 & $54 \cdot 3$ & 549 & 83.1 & 354 & 56.8 & 265 & 63.7 & 597 & $46 \cdot 1$ & & \\
\hline Income & & & & & & & & & & & & & 369.375 & $<0.001$ \\
\hline Poorest & 1234 & $23 \cdot 9$ & 373 & 19.0 & 303 & $42 \cdot 9$ & 155 & 23.0 & 85 & $19 \cdot 0$ & 318 & $23 \cdot 1$ & & \\
\hline Poor & 1157 & 22.4 & 352 & 18.0 & 209 & $29 \cdot 6$ & 182 & $27 \cdot 0$ & 133 & 29.8 & 281 & 20.4 & & \\
\hline Middle & 967 & $18 \cdot 7$ & 369 & $18 \cdot 8$ & 95 & 13.5 & 154 & $22 \cdot 8$ & 97 & 21.7 & 252 & $18 \cdot 3$ & & \\
\hline Richer & 989 & $19 \cdot 2$ & 448 & $22 \cdot 9$ & 60 & 8.5 & 98 & 14.5 & 86 & $19 \cdot 2$ & 297 & $21 \cdot 6$ & & \\
\hline Richest & 815 & $15 \cdot 8$ & 418 & 21.3 & 39 & 5.5 & 85 & $12 \cdot 6$ & 46 & $10 \cdot 3$ & 227 & $16 \cdot 5$ & & \\
\hline Breast-feeding during & e previc & day & & & & & & & & & & & $189 \cdot 362$ & $<0.001$ \\
\hline Yes & 2148 & $44 \cdot 4$ & 683 & 37.3 & 442 & 65.5 & 250 & 40.1 & 238 & 55.6 & 535 & $41 \cdot 8$ & & \\
\hline No & 2692 & $55 \cdot 6$ & 1150 & $62 \cdot 7$ & 233 & 34.5 & 373 & 59.9 & 190 & 44.4 & 746 & 58.2 & & \\
\hline
\end{tabular}

intake of dairy products in comparison to other ethnic groups (Tibetan $v$. Han $=75.1 v \cdot 62.9 \%, P<0.001$ ), but generally have a lower intake of legumes and nuts (Tibetan $v$. Han $=12 \cdot 3 v$. $26.4 \%, P<0.001$ ), eggs (Tibetan $v$. Han $=25.9$ v. $43.6 \%$, $P<0.001$ ), fruits and vegetables (Tibetan $v$. Han $=52.5 v \cdot 69.8 \%$, $P<0.001)$. After stratification by age groups, the consumption of all types of foods by different ethnic groups were higher among children aged 12-23 months as compared with children aged 6-11 months, except for the consumption of dairy products within the Yi and Uighur groups. Moreover, the Han children showed higher consumption rates of nearly every types of food and had more children achieved MDD than other ethnicities, in both the 6-11 and 12-23 months groups.

\section{Stunting}

As shown in Table 3, the overall prevalence of stunting in surveyed areas was $17 \cdot 8 \%$. The prevalence of stunting among children aged 12-23 months is nearly twice as much in comparison with children aged 6-11 months (21.8 v.11.1\%, adjusted $\mathrm{RR}=2 \cdot 10$ ). Young males appear to have a higher prevalence of stunting in comparison with their female counterparts $(20 \cdot 8 v$. $14.3 \%$, adjusted $R R=1.44$ ). Based on the multivariate analysis, elder aged males delivered at home and from a poor family with a low income were found to be significantly associated with the child's stunting. In addition, there were significant statistical associations between individual ethnic groups and stunting. The Yi, Tibetan and Uighur's children had 1.25, 1.29 and 1.72 times greater risk of stunting in contrast to children from the Han minority, respectively. Table 3 also examined the association between MDD and stunting. After adjusting for all other factors, children who did not achieve the MDD had a 1.15 times greater risk of stunting in comparison with those who received the recommended dietary intake. The gap of stunting prevalence among different ethnic groups was large. For the Han group, only $14.9 \%$ children were stunted, whereas the prevalence for the Yi, Tibetan and Uighur children were $26 \cdot 5,20 \cdot 3$ and $24.6 \%$, respectively.

\section{Discussion}

Timely and adequate management of complementary diets are necessary for infants aged 6 months and older; breast-feeding exclusively is insufficient to fulfil a child's nutrition requirements. However to date, socio-economics situations have been known to limit dietary diversity among children in China, especially for those who reside in poorer western regions. Our research showed that $44.5 \%$ of children aged $6-23$ months in 
Table 2. Relative risks of inadequate minimum dietary diversity among children aged 6-23 months in forty-two counties of Western China (2011) (Percentages, relative risks and $95 \%$ confidence intervals)

\begin{tabular}{|c|c|c|c|c|c|c|c|c|}
\hline Characteristics & $N$ & $n$ & $\%$ & $95 \% \mathrm{Cl}$ & Crude RR & $95 \% \mathrm{Cl}$ & Adjusted RR* & $95 \% \mathrm{Cl}$ \\
\hline Overall & 5169 & 2868 & $44 \cdot 5$ & $43 \cdot 2,45 \cdot 9$ & & & & \\
\hline \multicolumn{9}{|l|}{ Age of child (months) } \\
\hline $6-11$ & 1933 & 801 & $58 \cdot 6$ & $56 \cdot 4,60 \cdot 8$ & 1.62 & $1.52,1.71$ & 1.39 & $1.31,1.49$ \\
\hline $12-23$ & 3236 & 2067 & $36 \cdot 1$ & $34.5,37.8$ & 1 & & 1 & \\
\hline \multicolumn{9}{|l|}{ Sex of child } \\
\hline Male & 2732 & 1503 & $45 \cdot 0$ & $43 \cdot 1,46 \cdot 9$ & 1.01 & $0.95,1.08$ & 1.03 & $0.97,1.09$ \\
\hline Female & 2418 & 1350 & $44 \cdot 2$ & $42 \cdot 2,46 \cdot 1$ & 1 & & 1 & \\
\hline \multicolumn{9}{|l|}{ Birth order of child } \\
\hline 1 & 2682 & 1658 & $38 \cdot 2$ & $36 \cdot 3,40 \cdot 0$ & 1 & & 1 & \\
\hline 2 & 1637 & 865 & $47 \cdot 2$ & $44 \cdot 7,49.6$ & 1.23 & $1 \cdot 15,1.32$ & 1.09 & $1.02,1.17$ \\
\hline$\geq 3$ & 671 & 229 & $65 \cdot 9$ & $62 \cdot 3,69 \cdot 5$ & $1 \cdot 72$ & $1.60,1.85$ & 1.07 & $0.98,1.16$ \\
\hline \multicolumn{9}{|l|}{ Caregiver's education } \\
\hline Illiteracy & 1630 & 672 & 58.8 & $56 \cdot 4,61 \cdot 2$ & $2 \cdot 86$ & $2.08,3.93$ & $2 \cdot 12$ & $1.52,2.96$ \\
\hline Primary & 1445 & 820 & $43 \cdot 3$ & $40 \cdot 7,45 \cdot 8$ & $2 \cdot 10$ & $1.53,2.89$ & 1.72 & $1 \cdot 24,2 \cdot 40$ \\
\hline Secondary & 1932 & 1249 & $35 \cdot 4$ & $33 \cdot 2,37.5$ & $1 \cdot 72$ & $1 \cdot 25,2 \cdot 37$ & 1.47 & $1.06,2.05$ \\
\hline College and above & 151 & 120 & 20.5 & $14 \cdot 1,27 \cdot 0$ & 1 & & 1 & \\
\hline \multicolumn{9}{|l|}{ Ethnic group } \\
\hline Han & 1973 & 1284 & 34.9 & $32 \cdot 8,37 \cdot 0$ & 1 & & 1 & \\
\hline Yi & 717 & 193 & $73 \cdot 1$ & $69 \cdot 8,76 \cdot 3$ & 2.09 & $1 \cdot 94,2 \cdot 25$ & 1.15 & $1.04,1.28$ \\
\hline Tibetan & 674 & 329 & $51 \cdot 2$ & $47 \cdot 4,55 \cdot 0$ & 1.46 & $1.33,1.61$ & 1.09 & $0.99,1.21$ \\
\hline Uighur & 438 & 197 & $55 \cdot 0$ & $50 \cdot 4,59 \cdot 7$ & 1.57 & $1.42,1.74$ & 1.52 & $1.36,1.71$ \\
\hline Others & 1367 & 865 & $36 \cdot 7$ & $34 \cdot 2,39 \cdot 3$ & 1.05 & $0.95,1.15$ & 0.99 & $0.90,1.09$ \\
\hline \multicolumn{9}{|l|}{ Place of delivery } \\
\hline County hospital & 3118 & 1984 & $36 \cdot 4$ & $34 \cdot 7,38 \cdot 1$ & 1 & & 1 & \\
\hline Township hospital & 736 & 423 & 42.5 & $39 \cdot 0,46 \cdot 1$ & $1 \cdot 16$ & $1.06,1.28$ & 1.02 & $0.93,1.13$ \\
\hline At home & 1206 & 404 & $66 \cdot 5$ & $63 \cdot 8,69 \cdot 2$ & 1.82 & $1.71,1.94$ & $1 \cdot 30$ & $1 \cdot 20,1.41$ \\
\hline \multicolumn{9}{|l|}{ Income } \\
\hline Poorest & 1230 & 531 & $56 \cdot 8$ & $54 \cdot 1,59 \cdot 6$ & 1.86 & $1 \cdot 66,2.08$ & 1.32 & $1.17,1.50$ \\
\hline Poor & 1147 & 560 & $51 \cdot 2$ & $48 \cdot 3,54 \cdot 1$ & 1.67 & $1.49,1.88$ & 1.33 & $1.18,1.51$ \\
\hline Middle & 962 & 559 & 41.9 & $38 \cdot 8,45 \cdot 0$ & 1.37 & $1 \cdot 20,1.56$ & $1 \cdot 17$ & $1.03,1.33$ \\
\hline Richer & 983 & 636 & $35 \cdot 3$ & $32 \cdot 3,38 \cdot 3$ & $1 \cdot 15$ & $1.01,1.32$ & 1.05 & $0.91,1.20$ \\
\hline Richest & 813 & 565 & $30 \cdot 5$ & $27 \cdot 3,33 \cdot 7$ & 1 & & 1 & \\
\hline \multicolumn{9}{|c|}{ Breast-feeding during the previous day } \\
\hline Yes & 2136 & 835 & $60 \cdot 9$ & $58.8,63.0$ & 1.95 & $1.83,2.09$ & 1.55 & $1.44,1.66$ \\
\hline No & 2680 & 1846 & $31 \cdot 1$ & $29 \cdot 4,32 \cdot 9$ & 1 & & 1 & \\
\hline
\end{tabular}

* Adjusted for all the variables in Table 2 .
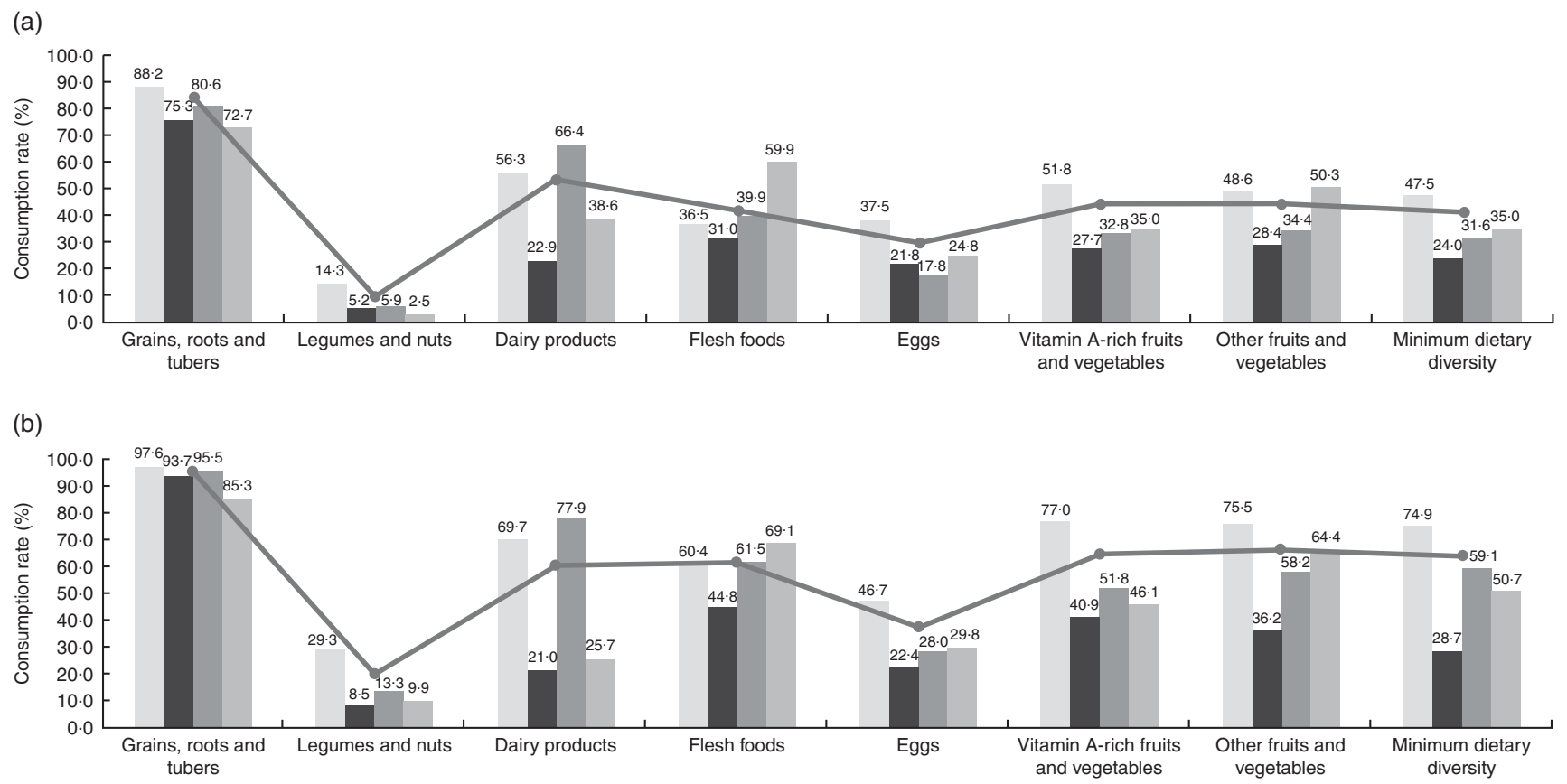

Fig. 1. Comparison of complementary food diversity among children aged (a) 6-11 months and (b) 12-23 months by ethnic groups. $\square$, Han; $\square$, Yi; $\square$, Tibetan; $\square$, Uighur; $-\mathrm{O}$, total. 
Table 3. Relative risks of stunting among children aged 6-23 months in forty-two counties of Western China (2011) (Percentages, relative risks and $95 \%$ confidence intervals)

\begin{tabular}{|c|c|c|c|c|c|c|c|c|}
\hline Characteristics & $N$ & $n$ & $\%$ & $95 \% \mathrm{Cl}$ & Crude RR & $95 \% \mathrm{Cl}$ & Adjusted RR* & $95 \% \mathrm{Cl}$ \\
\hline \multicolumn{8}{|l|}{ Age of child (months) } & \\
\hline $6-11$ & 1903 & 211 & $11 \cdot 1$ & $9 \cdot 7,12 \cdot 5$ & 1 & & 1 & \\
\hline $12-23$ & 3170 & 690 & $21 \cdot 8$ & $20 \cdot 3,23 \cdot 2$ & 1.96 & $1 \cdot 70,2 \cdot 26$ & $2 \cdot 10$ & $1.78,2.49$ \\
\hline \multicolumn{9}{|l|}{ Sex of child } \\
\hline Male & 2688 & 560 & $20 \cdot 8$ & $19 \cdot 3,22 \cdot 4$ & 1.45 & $1 \cdot 28,1.64$ & 1.44 & $1.26,1.64$ \\
\hline Female & 2385 & 341 & $14 \cdot 3$ & $12 \cdot 9,15 \cdot 7$ & 1 & & 1 & \\
\hline \multicolumn{9}{|l|}{ Birth order of child } \\
\hline 1 & 2636 & 438 & $16 \cdot 6$ & $15 \cdot 2,18 \cdot 0$ & 1 & & 1 & \\
\hline 2 & 1603 & 276 & $17 \cdot 2$ & $15 \cdot 4,19 \cdot 1$ & 1.03 & $0.90,1.18$ & 0.92 & $0.79,1.06$ \\
\hline$\geq 3$ & 663 & 164 & $24 \cdot 7$ & $21 \cdot 5,28 \cdot 0$ & 1.48 & $1 \cdot 27,1 \cdot 74$ & 0.98 & $0.81,1.18$ \\
\hline \multicolumn{9}{|l|}{ Caregiver's education } \\
\hline Illiteracy & 1595 & 344 & $21 \cdot 6$ & $19 \cdot 5,23 \cdot 6$ & $2 \cdot 71$ & $1.56,4.70$ & 1.75 & $0.95,3.24$ \\
\hline Primary & 1409 & 258 & $18 \cdot 3$ & $16 \cdot 3,20 \cdot 3$ & $2 \cdot 30$ & $1 \cdot 32,4.00$ & $1 \cdot 72$ & $0.93,3.16$ \\
\hline Secondary & 1908 & 285 & 14.9 & $13 \cdot 3,16 \cdot 5$ & 1.87 & $1.08,3.26$ & 1.58 & $0.86,2.90$ \\
\hline College and above & 151 & 12 & 7.9 & $3 \cdot 6,12 \cdot 3$ & 1 & & 1 & \\
\hline \multicolumn{9}{|l|}{ Ethnic group } \\
\hline Han & 1921 & 270 & $14 \cdot 1$ & $12 \cdot 5,15 \cdot 6$ & 1 & & 1 & \\
\hline Yi & 716 & 190 & $26 \cdot 5$ & $23 \cdot 3,29 \cdot 8$ & 1.88 & $1 \cdot 60,2 \cdot 22$ & 1.25 & $1.00,1.58$ \\
\hline Tibetan & 649 & 132 & $20 \cdot 3$ & $17 \cdot 2,23 \cdot 4$ & 1.44 & $1.19,1.74$ & 1.29 & $1.05,1.60$ \\
\hline Uighur & 439 & 108 & $24 \cdot 6$ & $20 \cdot 6,28 \cdot 6$ & $1 \cdot 75$ & $1 \cdot 43,2 \cdot 13$ & 1.72 & $1 \cdot 38,2 \cdot 15$ \\
\hline Others & 1348 & 201 & $14 \cdot 9$ & $13 \cdot 0,16 \cdot 8$ & 1.06 & $0.89,1.25$ & $1 \cdot 10$ & $0.92,1.32$ \\
\hline \multicolumn{9}{|l|}{ Place of delivery } \\
\hline County hospital & 3054 & 447 & $14 \cdot 6$ & $14 \cdot 1,16 \cdot 1$ & 1 & & 1 & \\
\hline Township hospital & 727 & 139 & $19 \cdot 1$ & $18 \cdot 3,22 \cdot 6$ & $1 \cdot 30$ & $1.09,1.55$ & $1 \cdot 16$ & $0.96,1.39$ \\
\hline At home & 1181 & 295 & $25 \cdot 0$ & $25 \cdot 9,29 \cdot 8$ & $1 \cdot 70$ & $1.49,1.94$ & $1 \cdot 23$ & $1.03,1.47$ \\
\hline \multicolumn{9}{|l|}{ Income } \\
\hline Poorest & 1196 & 259 & $21 \cdot 7$ & $19 \cdot 3,24 \cdot 0$ & $2 \cdot 13$ & $1.69,2.69$ & 1.56 & $1.19,2.03$ \\
\hline Poor & 1134 & 234 & $20 \cdot 6$ & $18 \cdot 3,23 \cdot 0$ & 2.03 & $1 \cdot 60,2.58$ & 1.58 & $1.22,2.06$ \\
\hline Middle & 947 & 167 & $17 \cdot 6$ & $15 \cdot 2,20 \cdot 1$ & $1 \cdot 74$ & $1 \cdot 35,2 \cdot 23$ & 1.47 & $1.12,1.93$ \\
\hline Richer & 967 & 148 & $15 \cdot 3$ & $13 \cdot 0,17 \cdot 6$ & 1.51 & $1.17,1.94$ & 1.38 & $1.05,1.82$ \\
\hline Richest & 800 & 81 & $10 \cdot 1$ & $8 \cdot 0,12 \cdot 2$ & 1 & & 1 & \\
\hline \multicolumn{9}{|c|}{ Breast-feeding during the previous day } \\
\hline Yes & 2111 & 374 & $17 \cdot 7$ & $16 \cdot 1,19 \cdot 3$ & 1.03 & $0.91,1.17$ & $1 \cdot 15$ & $0.99,1.32$ \\
\hline No & 2617 & 448 & $17 \cdot 1$ & $15 \cdot 7,18 \cdot 6$ & 1 & & 1 & \\
\hline \multicolumn{9}{|c|}{ Minimum dietary diversity } \\
\hline Received & 2778 & 433 & $15 \cdot 6$ & $14 \cdot 2,16 \cdot 9$ & 1 & & 1 & \\
\hline Not received & 2269 & 464 & $20 \cdot 4$ & $18 \cdot 8,22 \cdot 1$ & $1 \cdot 31$ & $1 \cdot 16,1.47$ & $1 \cdot 15$ & $1.01,1.31$ \\
\hline
\end{tabular}

* Adjusted for all the variables in Table 3.

the forty-two western counties failed to reach the MDD as defined by WHO. The adverse situation on dietary diversity from our study is similar to other surveys conducted within China's western areas ${ }^{(24,25)}$ and some undeveloped countries ${ }^{(26-28)}$. But the status was far worse as compared with those from the eastern regions (e.g. $60 \%$ for children aged 6 months and nearly $100 \%$ for children aged 12 months reported in Shanghai received $\mathrm{MDD}^{(29)}$ ) and central areas (e.g. reported from the poor rural counties in Hunan Province, $73.9 \%$ of the children aged $6-23$ months received $\mathrm{MDD}^{(30)}$ ) in China; this stark difference is indicative of a serious problem involving dietary diversity for children in Western China.

From the multivariable analysis results, socio-economic factors such as caregivers with low education level, low family income and children delivered at home were found to have greater risks of an inadequate MDD; these findings may suggest the importance of education, socio-economic status and access to health care services in relation to proper feeding practice. The sex of children' had insignificant impact on the adequacy of dietary diversity, this observation was consistent with most other studies $^{(24,26,31)}$. In addition, children with a higher birth order were less likely to have a diversified feeding pattern which may indicate a negligence on a child's individual care when parents have more than one child.

One highlight of our study were the stark differences and similarities observed based on feeding patterns by different ethnicities. First, minority groups showed a greater dietary disparity in comparison with the Han group. Nearly two-thirds of the Han's children met the MDD criteria; this contrasts with the only one-fourth of the Yi's, about half of the Uighur, and Tibetan children who met the similar criteria. Furthermore, we observed that children from various ethnic groups showed different patterns of complementary food in addition to a standardised diet. For all the studied ethnic groups, grains, roots and tubers comprised predominant proportion of their diets, but legumes and nuts are rarely chosen. Due to the high altitude and barren soil, most Tibetans lived on a grazing based lifestyle; therefore, groups within this population rely more on meat and dairy products as a common food source within their diets, rather than vegetable and fruits. Although meat and dairy food can provide protein, abundant energy content, and micro-elements such as Fe and $\mathrm{Zn}$, there is a greater need for 
caregivers to be more careful with these sorts of foods and personal hygiene, which may be contaminated at the point of source. The food structure of Uighur children is most comparable with Uighur adults; this would include a greater intake of flesh foods, but less eggs, dairy products, vitamin A-rich fruits and vegetables intake. As previously mentioned meat may provide essential amino acids, additionally the increase of dietary cholesterol as found in eggs and dairy products is noticeable; the high protein and high energetic intake maintained in adult diets may lead to unbalanced growth or development in children. On the other hand, dietary patterns among ethnic groups were also shown to be subject to modification by age groups; older children are more likely to be exposed to a greater diversity of food types in comparison to their younger counterparts. For children under 1 year of age, those in Han group showed a richer diversity of complementary foods types; these children are more likely to achieve MDD earlier than other groups, this observation may account for lower stunting prevalence within this ethnic group.

Among all the groups studied, the Yi minority showed greatest disparity in nearly every type of food group. One possible explanation for this phenomenon may be due to both limited access to resources and local traditional customs. Most of the Yi people in our study lived in rural mountainous areas with inconvenient traffic infrastructure. The local average household income was low and most residence lived on coarse grains (as other food resources were limited) ${ }^{(32,33)}$. Lack of sufficient food supplies and proper feeding knowledge, parents may tend to feed their children only with breast milk and grain foods. Further interventions should focus on improving Yi's simple food structure to further develop feeding practice education specifically for this minorities.

Stunting reflects chronic undernutrition during the 'critical window' of growth and development for both infants and young children. The overall stunting prevalence among surveyed subjects living in Western China in our study was $17 \cdot 8 \%$; this was higher than the national average prevalence of rural children of $11 \cdot 2 \%$ and mostly similar to $18.7 \%$ in poor rural areas in $2013^{(34)}$. Past evidence has illustrated that a lack of food dietary diversity will accumulate to child's malnutrition status, especially stunting ${ }^{(35-37)}$, these findings have also been confirmed in our multivariate analysis. A study in India has showed a non-significant difference of stunting prevalence between children with medium and low food diversity, however, the difference became significant when comparing children with high and low food diversity ${ }^{(38)}$. Male's sex was more prevalent in stunting, but this was not observed in complementary food diversity. The high stunting prevalence for male children was consistent with previous studies $^{(7,15,38)}$ and with the high under 5-year mortality rate of boys in China ${ }^{(1)}$. Education level and income were found significantly associated with MDD, whereas only income was associated with stunting. The significant association between education and stunting in the binary model disappeared after adjusting for MDD and other variables; this indicates that education levels of caregivers may be only the direct causal factor of MDD, but not stunting (education level $\rightarrow$ MDD $\rightarrow$ stunting). Other literatures also observed the association between a caregiver's education and stunting when the authors did not adjust for dietary diversity ${ }^{(15,36)}$. However, the relationship between income and stunting remains significant in both the binary and multivariable models, these observations suggest that income may confound the relationship of MDD and stunting (income $\rightarrow$ MDD $\rightarrow$ stunting, income $\rightarrow$ stunting). In another way, income is an independent risk factor which can be mediated to reduce stunting in children.

In addition, the gaps of stunting prevalence among different ethnic groups was large. Of the three-major minority ethnic groups investigated (Yi, Tibetan, Uygur), these groups showed higher stunting prevalence in comparison to the Han group. In particular, the prevalence of stunting within the Yi group was as high as $26.5 \%$, which was comparable with the level of Southern Africa ${ }^{(39)}$. As shown in Table 1, different ethnic groups have variable distributions of children's sex, birth order, caregiver's education, delivery place, postpartum visit, income and breast-feeding status. Among all these factors above, children's sex, birth order, caregiver's education level, place of delivery, income and breast-feeding status are found to be statistically associated with MDD; whereas place of delivery, income and MDD are associated with stunting. Therefore, the disparity among different ethnic groups may be partly accounted for by the inequalities of socio-economic factors and complementary dietary performance among different ethnic groups.

Several limitations of our study should be considered. First, the tool we used to assess dietary diversity of children is 24-h dietary recall; whereas this method is rather convenient for collecting data, this conservative approach may affect our information on the food diversity based on caregiver recall bias. In addition, the types of food consumed by children may vary from day by day, therefore the results of the survey in 24 hs may differ from the actual dietary situation. Second, our study only focused on types of complementary foods, rather than the meal frequency, food quantity, food sanitation or caregiver hygiene; other feeding practice factors which are also key indicators of child growth and may play roles in developing sufficient interventions to reduce stunting prevalence. One important strength of our study is that there is a scarcity of literature that focus on dietary diversity issues in Western China; our novel results may contribute to current evidence of associations between dietary diversity and the stunting of children within western rural areas.

To conclude, our study found that nearly $44.5 \%$ complementary feeding diversity has not met the WHO recommended MDD; an overall $17.8 \%$ stunting rate is existent in the poor counties of Western China. Inadequate MDD was found to be positively associated with childhood stunting. Moreover, different ethnicities showed greater variation in achieving MDD and should be further explored in future research to benefit individual groups as well as the general health of Western China. To halve the stunting prevalence by 2025, further efforts are needed: for regions with limited food sources, supplementary nutrition programmes should be conducted; for these ethnic minority groups, health education for caregivers as well as nutrition interventions focusing on feeding practices in tandem with local culture and food availability improvement should be addressed. 


\section{Acknowledgements}

The authors would like to thank research teams from Lanzhou University, Guangxi Medical University and Chongqing Medical University for their hard work in orchestrating the field work and identifying study population. The authors also want to thank all of family members who participated in this study from the forty-two counties in Western China.

This work was funded by a grant from UNICEF China (YH702).

H. Z. and A. W. formulated the research question and the first draft of the article and analysed the data. Y. W., H. Z., R. W. S., X. H., S. G. and Y. Y. designed study and carried it out. J. J.-S. and C. M. proofread and polished the language of this article. Y. W. and H. Z. provided overall guidance to the study. All authors contributed to the writing and revision of the article.

The authors declare that there are no conflicts of interest.

\section{References}

1. Wang Y, Li X, Zhou M, et al. (2016) Under-5 mortality in 2851 Chinese counties, 1996-2012: a subnational assessment of achieving MDG 4 goals in China. Lancet 387, 273-283.

2. United Nations International Children's Emergency Fund, World Health Organization, World Bank, et al. (2015) Levels and Trends in Child Mortality. Report 2015. New York: UNICEF, WHO, The World Bank, United Nations Population Division.

3. Independent Expert Review Group (2014) Every Woman, Every Child: A Post-2015 Vision. The Third Report of the Independent Expert Review Group on Information and Accountability for Women's and Children's Health. Geneva: WHO.

4. United Nations (2016) Goal 2: end hunger, achieve food security and improved nutrition and promote sustainable agriculture. http://www.un.org/sustainabledevelopment/hunger/ (accessed June 2016).

5. Ruyter DJ (2012) On optimal development and becoming an optimiser. J Philos Educ 46, 25-41.

6. Black MM, Walker SP, Fernald LCH, et al. (2017) Early childhood development coming of age: science through the life course. Lancet 389, 77-90.

7. Black RE, Victora CG, Walker SP, et al. (2013) Maternal and child undernutrition and overweight in low-income and middle-income countries. Lancet 382, 427-451.

8. World Health Organization (2014) Comprehensive Implementation Plan on Maternal, Infant and Young Child Nutrition. Geneva: WHO.

9. World Health Organization (2017) WHO Global Database on Child Growth and Malnutrition. Stunting: 1990-2025. http:// www.who.int/nutgrowthdb/en/ (accessed October 2017).

10. Martorell R \& Young MF (2012) Patterns of stunting and wasting: potential explanatory factors. Adv Nutr 3, 227-233.

11. Bhutta ZA, Das JK, Rizvi A, et al. (2013) Evidence-based interventions for improvement of maternal and child nutrition: what can be done and at what cost? Lancet 382, 452-477.

12. Correia LL, Silva ACe, Campos JS, et al. (2014) Prevalence and determinants of child undernutrition and stunting in semiarid region of Brazil. Rev Saúde Pública 48, 19-28.

13. Dang S, Yan H \& Wang D (2014) Implication of World Health Organization growth standards on estimation of malnutrition in young Chinese children: two examples from rural western China and the Tibet region. J Child Health Care 18, 358-368.
14. Dang S, Yan H \& Yamamoto S (2008) High altitude and early childhood growth retardation: new evidence from Tibet. EurJ Clin Nutr 62, 342-348.

15. Jiang Y, Su X, Wang C, et al. (2015) Prevalence and risk factors for stunting and severe stunting among children under three years old in mid-western rural areas of China. Child Care Health Dev 41, 45-51.

16. Wu L, Yang Z, Yin SA, et al. (2015) The relationship between socioeconomic development and malnutrition in children younger than 5 years in China during the period 1990 to 2010. Asia Pac J Clin Nutr 24, 665-673.

17. Bianba B, Yangzong Y, Gonggalanzi G, et al. (2015) Anthropometric measures of 9- to 10-year-old native Tibetan children living at 3700 and $4300 \mathrm{~m}$ above sea level and Han Chinese living at $3700 \mathrm{~m}$. Medicine (Baltimore) 94, e1516.

18. Xu F, Binns C, Nazi G, et al. (2006) A comparison of breastfeeding among Han, Uygur and other ethnic groups in Xinjiang, PR China. BMC Public Health 6, 196.

19. Harris NS, Crawford PB, Yangzom Y, et al. (2001) Nutritional and health status of Tibetan children living at high altitudes. $N$ Engl J Med 344, 341-347.

20. National Bureau of Statistics of China (2010) National data annual. http://data.stats.gov.cn/english/easyquery.htm?cn=C01 (accessed April 2016).

21. United Nations International Children's Emergency Fund (2009) Multiple Indicator Cluster Survey (MICS). http://www. unicef.org/statistics/index_24302.html (accessed May 2016).

22. World Health Organization Multicentre Growth Reference Study Group (2006) WHO Child Growth Standards: Length/ Height-for-Age, Weight-for-Age, Weight-for-Length, Weight-forHeight and Body Mass Index-for-Age: Methods and Development. Geneva: WHO.

23. World Health Organization, United Nations International Children's Emergency Fund, United States Agency for International Development, et al. (2008) Indicators for assessing infant and young child feeding practices. http://www.who.int/nutrition/ publications/infantfeeding/9789241596664/en/ (accessed January 2017).

24. Hipgrave DB, Fu X, Zhou $\mathrm{H}$, et al. (2014) Poor complementary feeding practices and high anaemia prevalence among infants and young children in rural central and western China. Eur J Clin Nutr 68, 916-924.

25. Ban L, Guo S, Scherpbier RW, et al. (2017) Child feeding and stunting prevalence in left-behind children: a descriptive analysis of data from a central and western Chinese population. Int J Public Health 62, 143-151.

26. Joshi N, Agho KE, Dibley MJ, et al. (2012) Determinants of inappropriate complementary feeding practices in young children in Nepal: secondary data analysis of Demographic and Health Survey 2006. Matern Child Nutr 8, Suppl. 1, 45-59.

27. Saaka M, Wemakor A, Abizari AR, et al. (2015) How well do WHO complementary feeding indicators relate to nutritional status of children aged 6-23 months in rural Northern Ghana? BMC Public Health 15, 1157.

28. Beyene M, Worku AG \& Wassie MM (2015) Dietary diversity, meal frequency and associated factors among infant and young children in Northwest Ethiopia: a cross-sectional study. BMC Public Health 15, 1007.

29. Woo JG, Herbers PM, McMahon RJ, et al. (2015) Longitudinal development of Infant complementary diet diversity in 3 international cohorts. J Pediatr 167, 969-974.e961.

30. Zhou X, Fang JQ, Luo JY, et al. (2017) [Status of, and factors associated with, complementary feeding among infants and young children aged 6-23 months in poor rural areas of Hunan Province, China]. Zhonghua Yu Fang Yi Xue Za Zhi 51, 58-64. 
31. Liu S, Li J, Gong C, et al. (2014) [Evaluation of the feeding status of infants and young children under 2 years old in rural areas of Hubei province]. Zhonghua Yu Fang Yi Xue Za Zhi 48, 705-709.

32. Gao Y, Ran X-W, Xie X-H, et al. (2011) Prevalence of overweight and obesity among Chinese Yi nationality: a cross-sectional study. BMC Public Health 11, 919.

33. Zhou J, Huang C, Xu Y, et al. (2003) [The dietary patterns and nutritional status of adult Yi people in Liangshan autonomous region]. Wei Sheng Yan Jiu 32, 246-248.

34. Yu DM, Zhao LY, Yang ZY, et al. (2016) Comparison of Undernutrition Prevalence of Children under 5 Years in China between 2002 and 2013. Biomed Environ Sci 29, 165-176.

35. Arimond M \& Ruel MT (2004) Dietary diversity is associated with child nutritional status: evidence from 11 demographic and health surveys. J Nutr 134, 2579-2585.
36. Mallard SR, Houghton LA, Filteau S, et al. (2014) Dietary diversity at 6 months of age is associated with subsequent growth and mediates the effect of maternal education on infant growth in urban Zambia. J Nutr $\mathbf{1 4 4}$, 1818-1825.

37. Marriott BP, White A, Hadden L, et al. (2012) World Health Organization (WHO) infant and young child feeding indicators: associations with growth measures in 14 low-income countries. Matern Child Nutr 8, 354-370.

38. Fenske N, Burns J, Hothorn T, et al. (2013) Understanding child stunting in India: a comprehensive analysis of socio-economic, nutritional and environmental determinants using additive quantile regression. PLOS One 8, e78692.

39. de Onis M, Blossner M \& Borghi E (2012) Prevalence and trends of stunting among pre-school children, 1990-2020. Public Health Nutr 15, 142-148. 